\title{
Implementation of structured ward round pro formas on an acute frailty unit
}

\author{
Authors: Sam Kleeman, Zainab Khan, Joanna Brecher, MD Mofijur Rahman, Nihethana Jegatheeswaran, \\ Hannah Costelloe, Sharose Shiraz, Christopher Chung, Jonathan Graff and Benjamin Jacobs
}

\section{Introduction}

Guidance from the Royal College of Physicians and the National Institute for Health and Care Excellence (NICE) recommends the use of structured approaches to ward rounds. There is evidence that such approaches increase staff satisfaction, reduce adverse events and reduce length of stay. The aim of this project was to implement a structured approach to ward rounds in an acute frailty unit at a busy district general hospital in the UK.

\section{Materials and methods}

Using a Plan, Do, Study, Act (PDSA) approach, a structured ward round pro forma was implemented over a 4 -week pilot period. Each week, we reviewed notes of 10 randomly selected patients to monitor adoption of the pro forma as well as utilisation of specific pro forma components (safety checklist, Gold Standard Framework (GSF) status, treatment escalation plan (TEP)). We used this data alongside feedback from the multidisciplinary team (MDT) to iteratively improve the pro forma design. MDT members were sent a survey to assess perception of the ward round documentation before and after the pilot period.

\section{Results and discussion}

Baseline survey data $(n=13)$ showed that MDT members felt that clear ward round documentation was important for the functioning of the MDT $(9.46 \pm 0.32 / 10)$ and that existing documentation was poor $(5.0 \pm 0.58 / 10) .13$ out of 13 responses $(100 \%)$ indicated that they felt a structured ward round pro forma would improve the quality of ward round documentation. At week 1, pro forma adoption rate was $50 \%$ (5/10), of which $40 \%$ (2/5), $60 \%$ (3/5) and $60 \%(3 / 5)$ included use of safety checklist, GSF status and TEP respectively. At week 2, adoption rate was $100 \%$ (10/10), of which $50 \%(5 / 10), 70 \%(7 / 10)$ and $40 \%(4 / 10)$ included use of safety checklist, GSF status and TEP respectively. At week 3, adoption rate was $100 \%$ (10/10), of which $70 \%(7 / 10), 80 \%$ (8/10) and $80 \%(8 / 10)$ included use of safety checklist, GSF status and TEP respectively. Results from a repeat survey of MDT members are awaited.

Authors: Barking, Havering and Redbridge University Hospitals NHS Trust

\section{Conclusion}

There is evidence of an unmet need for improved ward round documentation in our acute frailty unit. In this setting, it is feasible to introduce and rapidly adopt a structured ward round pro forma reflective of national guidelines. We aim to institute sustainable change through ongoing education and development of an electronic record-based pro forma.

\section{References}

1 Royal College of Physicians. Ward rounds in medicine. London: RCP, 2015. www.rcplondon.ac.uk/projects/outputs/ward-rounds-medicineprinciples-best-practice [Accessed 5 February 2019].

2 National Institute for Health and Care Excellence. Emergency and acute medical care in over 16s: service delivery and organisation (NG94). London: NICE, 2018. www.nice.org.uk/guidance/ng94/ chapter/Recommendations [Accessed 5 February 2019]. 